\title{
Loss of caveolin-1 promotes endothelial-mesenchymal transition during sepsis: A membrane proteomic study
}

\author{
XIAOBO HUANG, LINGAI PAN, HONG PU, YIPING WANG, XIAOQIN ZHANG, \\ CHUNLING LI and ZHENGLIN YANG
}

\begin{abstract}
Sichuan Academy of Medical Sciences and Sichuan Provincial People's Hospital, Chengdu, Sichuan 610072, P.R. China
\end{abstract}
Received October 22, 2012; Accepted December 21, 2012

DOI: $10.3892 / \mathrm{ijmm} .2013 .1432$

\begin{abstract}
Sepsis is a clinical syndrome that reflects an uncontrolled systemic inflammatory response to microbial infections. Endothelial cells, which are highly responsive to their extracellular environment, are the primary targets of sepsis-induced damage. Endothelial-mesenchymal transition (EndMT), characterized by loss of endothelial cell markers and gain of mesenchymal cell markers, contributes to embryonic cardiac formation as well as development of various diseases. We showed that incubation with septic serum induced a rapid loss of endothelial barrier integrity in a human umbilical vein endothelial cell (HUVEC) monolayer. Notably, exposure to septic serum triggered EndMT in HUVECs, companied by increased cell motility and invasion. Furthermore, membrane proteomic analysis was applied to analyze the key mediators in septic serum-induced EndMT. A total of 29 proteins with altered expression level were positively identified. Expression of four proteins with the most significant alteration, including caveolin-1, S100A4, $\alpha$-enolase and galectin-1, were validated by western blotting. Finally, we showed that restoration of caveolin-1 expression markedly attenuated septic serum-induced EndMT in HUVEC cells. This is the first report showing that endothelial cells undergo EndMT during sepsis. The present study may lead to a better understanding of the biological role of endothelial cells and caveolin-1 during sepsis.
\end{abstract}

\section{Introduction}

Sepsis, which reflects an uncontrolled systemic inflammatory response, is among the most significant challenges in critical care, with an estimated incidence of nearly 800,000 cases/year, and a mortality rate of $25 \%$, and up to $60 \%$ when shock is present (1). This syndrome usually includes at least two of the four signs of a systemic inflammatory response syndrome (SIRS): fever or

Correspondence to: Dr Zhenglin Yang, Sichuan Academy of Medical Sciences and Sichuan Provincial People's Hospital, Chengdu, Sichuan 610072, P.R. China

E-mail: yangzhenglin2012@126.com

Key words: sepsis, endothelial-mesenchymal transition, human umbilical vein endothelial cells, endothelial barrier, caveolin-1 hypothermia, tachycardia, rapid breathing, hyperleukocytosis or leukopenia (2). The pathophysiology of sepsis involves dysfunctions of complex interactions of signal pathways and impairment in host responses to pathogen-associated molecular patterns, resulting in chaos in inflammatory cytokine production, adhesion molecule expression, respiratory burst, and production of reactive nitrogen species (3).

Endothelial cells, which are highly responsive to their extracellular environment, are the primary targets of sepsisinduced damage (4). Exposure to septic serum, which contains lipopolysaccharide and abnormal levels of cytokines, chemokines and irons, lead to extensive endothelial dysfunction, manifested by a shift from the hemostatic balance towards a procoagulant phenotype, elevated leukocyte adhesion and abnormal regulation of vasomotor tone (5). Notably, it is known that progressive subcutaneous and body-cavity edema, usually leading to impairment of organ function, typically develops in patients with sepsis, suggesting wide-spread increases in vascular permeability (6). Although increased endothelial permeability has long been considered as a critical step in sepsis-associated organ failure, the underlying molecular mechanisms remain relatively unknown.

Endothelial-mesenchymal transition (EndMT), similar to epithelial-mesenchymal transition, is characterized by loss of endothelial cell markers, such as VE-cadherin and platelet endothelial cell adhesion molecule-1 (PECAM-1, also known as CD31), and gain of mesenchymal markers, such as $\alpha$-smooth muscle actin ( $\alpha$-SMA) and vimentin (7). During EndMT, resident endothelial cells delaminate from a polarized cell layer and migrate into the underlying tissue (8). Although EndMT is considered as an essential mechanism of endocardial cushion formation during cardiac and pulmonary development (9), recently, accumulating evidence has linked EndMT to various diseases. It is reported that loss of cell-cell junctions and acquisition of invasive and migratory properties during EndMT have also been linked to disruption of the endothelial barrier (10). Furthermore, a great portion of fibroblasts in rodent models of renal diseases was found to co-express the endothelial marker CD31 and the fibroblast/myofibroblast markers, fibroblast-specific protein-1 and/or $\alpha$-SMA (11). Furthermore, a significant number of fibroblasts raised from bleomycin-induced pulmonary fibrosis were of endothelial origin (12). However, whether EndMT occurs during sepsis is relatively unknown. 
Membrane-associated proteins, either embedded in the lipid bilayers or anchored to the membrane, possess multiple functions, including signal transduction, protein or iron transport, cytoskeleton assembly and cell adhesion (13). 2-DE-based proteomic approaches enable identification of alterations in membrane protein expression, allowing proteome-wide profiling of cellular responses upon stress (14). In this study, barrier integrity of a HUVEC monolayer was rapidly decreased by incubation with serum derived from sepsis patients. Notably, exposure to septic serum induced clear EndMT in HUVECs. Furthermore, proteomic analysis was introduced to screen for cellular alterations underlying septic serum-mediated EndMT, and caveolin-1 was identified as a key factor.

\section{Materials and methods}

Ethics statement. The study was approved by the Institutional Ethics Committee of Sichuan Academy of Medical Sciences and Sichuan Provincial People's Hospital. All participants provided written informed consent prior to sampling.

Clinical samples. The blood samples of sepsis patients and normal healthy donors were collected from Sichuan Provincial People's Hospital. Sepsis was diagnosed according to the criteria of the American College of Chest Physicians/Society of Critical Care Medicine (25). The blood samples were centrifuged at $900 \mathrm{x} \mathrm{g}$ for $10 \mathrm{~min}$ at room temperature, and the cell-free supernatants were stored immediately in aliquots at $-80^{\circ} \mathrm{C}$ until use.

Cell culture and septic serum treatment. Human umbilical vein endothelial cells (HUVECs) were purchased from ATCC and were maintained in Dulbecco's modified Eagle's medium (DMEM; Gibco, USA) containing 10\% fetal calf serum (Gibco, USA), penicillin (100 U/l) and streptomycin (10 mg/l). Cells were incubated in a humidified atmosphere containing $5 \% \mathrm{CO}_{2}$ at $37^{\circ} \mathrm{C}$ and passaged every 5 days at a split ratio of 1:4 using trypsin. For assessment of the effect of septic serum on endothelial cells, confluent HUVECs were incubated with DMEM medium containing $20 \%$ septic serum for the indicated time. Those HUVECs treated with DMEM medium containing $20 \%$ healthy serum were used as the control group.

Determination of endothelial barrier dysfunction. HUVECs were seeded on $0.4-\mathrm{mm}$ pore size culture inserts and were cultured until confluence was reached. The cells were treated with the medium containing septic serum for the indicated time, and the medium was replaced with fresh medium containing blue dextran dye $(2.3 \mathrm{mg} / \mathrm{ml})$. Fifty milliliters of basolateral medium was sampled after $12 \mathrm{~h}$ of incubation, and was measured using a microplate reader (Safire; Tecan, Morrisville, NC, USA) at $610 \mathrm{~nm}$.

Cell migration and invasion assays. In vitro cell migration and invasion assays were performed using Transwell 24-well chambers (Corning). Cells were seed in the upper chamber. For monitoring cell invasion, the upper side of the filter was pre-covered with Matrigel (Collaborative Research, Inc., Boston, MA, USA). After $12 \mathrm{~h}$ for the migration assay or $24 \mathrm{~h}$ for the invasion assay, cells on the upper side of the filter were removed and those cells on the underside of the filter were stained with crystal violet, and counted with an inverted microscope (Zeiss Axiovert).

Immunocytofluorescence. Cells were washed with phosphatebuffered saline (PBS) ( $\mathrm{pH} 7.4$ ) three times and fixed in $4 \%$ paraformaldehyde at room temperature for $10 \mathrm{~min}$. After washing three times in PBS, the slides were incubated with $3 \%$ bovine serum albumin for $30 \mathrm{~min}$ and then incubated with the primary antibodies for $2 \mathrm{~h}$ at room temperature. After washing for three times, the slides were incubated with secondary antibody conjugated to TRITC for $30 \mathrm{~min}$ at room temperature. The specimens were analyzed via a fluorescence microscope.

Membrane protein purification and 2-DE analyses. Cells $\left(10^{9}\right)$ were harvested and re-suspended in homogenization buffer (50 mM HEPES, pH 7.4, $1 \mathrm{mM} \mathrm{CaCl}_{2}, 1 \mathrm{mM}$ EDTA, $1 \mathrm{mM}$ vanadate, and $1 \mathrm{mM}$ phenylmethylsulfonyl fluoride). After cells were broken down using a Dounce homogenizer (Wheaton), the cell samples were centrifuged at 3,000 x g for $10 \mathrm{~min}$ at $4^{\circ} \mathrm{C}$ to remove nuclei. The supernatant was then mixed with $80 \%(\mathrm{w} / \mathrm{v})$ sucrose, and overlaid with sucrose at gradient concentrations, including 35, 30, 25, 20, 15, 10 and $5 \%$. The gradients were centrifuged at 200,000 x g for $24 \mathrm{~h}$ at $4^{\circ} \mathrm{C}$. After centrifugation, two bands that appeared in the upper middle region of the tube were collected, diluted in PBS, and centrifuged for 166,000 x g for an additional $2 \mathrm{~h}$ at $4^{\circ} \mathrm{C}$. The pellets were suspended in lysis buffer ( $7 \mathrm{M}$ urea, $2 \mathrm{M}$ thiourea, 4\% CHAPS) containing protease inhibitor mixture 8340 (Sigma). Protein concentrations were determined using the DC protein assay kit. Protein samples were applied to IPG strips (all were from Bio-Rad) for $16 \mathrm{~h}$ of rehydration. After rehydration, the strips were submitted to IEF, and then equilibrated in equilibration buffer I (containing $130 \mathrm{mM}$ DTT) and II (containing $200 \mathrm{mM}$ iodoacetamide) for $15 \mathrm{~min}$, respectively. The second dimension was performed using $12 \%$ SDS-PAGE, and the gels were stained using CBB R-250 (Merck). The gels were scanned using the Bio-Rad GS-800 scanner $(400-750 \mathrm{~nm})$, and alterations in protein spots were analyzed using PDQuest 2-D analysis software (Bio-Rad). In-gel digestion of proteins was carried out using mass spectrometry grade Trypsin Gold (Promega, Madison, WI, USA) according to the manufacturer's instructions. Mass spectrum analysis was performed using a Q-TOF mass spectrometer fit with an ESI source (Waters). The MS/MS data were processed with MassLynx v. 4.1 software (both were from Micromass, Manchester, UK) which converted MS/MS data into PKL files. The PKL files were analyzed using the online Mascot search engine (http://www.matrixscience.com).

Western blotting. Cell samples were lysed with RIPA buffer and quantified by the DC protein assay kit (Bio-Rad). Protein samples were separated by $12 \%$ SDS-PAGE and transferred to PVDF membranes (Millipore). The membranes were incubated with $5 \% \mathrm{FBS}$ overnight at $4^{\circ} \mathrm{C}$, and subsequently probed with primary antibodies: mouse anti-caveolin-1 (diluted 1:1,000; Santa Cruz Biotechnology, Inc.), rabbit anti-galectin-1 (diluted 1:1,000), rabbit anti- $\alpha$-enolase (diluted 1:2,000), rabbit anti-S100A4 (diluted 1:2,000) (all were from Abcam). After washing for three times, the membranes were then incubated with secondary antibody conjugated to horseradish peroxidase 
Table I. Clinical parameters of sepsis patients.

\begin{tabular}{|c|c|c|c|c|c|c|c|c|}
\hline $\begin{array}{l}\text { Patient } \\
\text { no. }\end{array}$ & $\begin{array}{c}\text { Age } \\
\text { (years) }\end{array}$ & $\begin{array}{c}\text { APACHEII } \\
\text { score }\end{array}$ & $\begin{array}{l}\text { WBC } \\
\left(10^{9} / 1\right)\end{array}$ & $\mathrm{N}$ & $\begin{array}{c}\mathrm{Cr} \\
(\mu \mathrm{mol} / 1)\end{array}$ & $\begin{array}{c}\text { PCT } \\
(\mathrm{ng} / \mathrm{ml})\end{array}$ & $\begin{array}{c}\text { EM } \\
\text { (years) }\end{array}$ & Prognosis \\
\hline 1 & 32 & 22 & 18.34 & 78 & 233.6 & 18.1 & 13.9 & Death \\
\hline 2 & 43 & 17 & 24.5 & 93 & 638.9 & 180.1 & 21.5 & Discharge \\
\hline 3 & 57 & 32 & 1.05 & 82 & 294.7 & 49.5 & 72 & Death \\
\hline 4 & 64 & 22 & 19.34 & 87 & 211.3 & 34.6 & 23.1 & Discharge \\
\hline 5 & 63 & 13 & 6.92 & 89 & 238.2 & 23.7 & 21.8 & Death \\
\hline 6 & 71 & 13 & 14.97 & 81 & 86.2 & 26.1 & 11 & Discharge \\
\hline 7 & 67 & 39 & 5.72 & 85 & 165 & 21.1 & 23 & Death \\
\hline 8 & 73 & 13 & 2.8 & 79 & 52.6 & 34 & 10 & Discharge \\
\hline 9 & 79 & 15 & 5.88 & 83 & 63.7 & 32 & 15 & Death \\
\hline 10 & 73 & 21 & 15.11 & 99 & 52.9 & 26.5 & 23 & Death \\
\hline 11 & 72 & 21 & 9.52 & 90 & 160.9 & 2.14 & 22 & Discharge \\
\hline 12 & 81 & 39 & 4.13 & 80 & 650.5 & $>200$ & 73 & Death \\
\hline 13 & 64 & 19 & 18.12 & 92 & 213.4 & 43.3 & 21 & Discharge \\
\hline 14 & 71 & 23 & 21.34 & 87 & 198.2 & 32.1 & 19 & Discharge \\
\hline 15 & 63 & 21 & 25.42 & 89 & 122.4 & 33 & 16 & Discharge \\
\hline 16 & 81 & 27 & 24.22 & 79 & 109.8 & 24 & 67 & Death \\
\hline 17 & 71 & 35 & 22.11 & 92 & 215.4 & 22.5 & 78 & Death \\
\hline
\end{tabular}

WBC, white blood cell count; N, percentage of neutrophils; Cr, creatinine; PCT, procalcitonin; EM, expected mortality.

(diluted 1:10,000; Santa Cruz Biotechnology, Inc.) for $2 \mathrm{~h}$ at room temperature. Blots were visualized using enhanced chemiluminescence reagents (Amersham Biosciences). $\beta$-actin was used as an internal control.

Statistics analysis. Differences between two groups were assessed by the Student's t-test at $\mathrm{P}<0.05 ; \mathrm{P}<0.01$; or $\mathrm{P}<0.001$.

\section{Results}

Septic serum decreases endothelial barrier integrity. Previous studies have shed light on the loss of endothelial barrier integrity under sepsis (15); however, the molecular mechanisms underlying this process are relatively unknown. As an initial test, we sought to determine whether incubation with the serum derived from sepsis patients could alter endothelial barrier integrity. To this end, HUVECs were used as an in vitro model, and septic serum was obtained from 17 sepsis patients. The detailed information of these patients is listed in Table I. As results, exposure to septic serum for $12 \mathrm{~h}$ resulted in a significant decrease in HUVEC monolayer integrity (Fig. 1).

Septic serum induces EndMT in HUVECs. To elucidate septic serum-induced leakage in HUVEC monolayer, we determined the vitality of HUVECs exposed to septic serum. However, by TUNEL assay, no difference in the number of apoptotic cells was found between the septic serum-treated cells and untreated control after a 12-h incubation, although an increased cell death was noted after $36 \mathrm{~h}$ of incubation (data not shown). These observations suggest that endothelial barrier dysfunction induced by a 12-h exposure to septic serum may not be due to endothelial cell death.

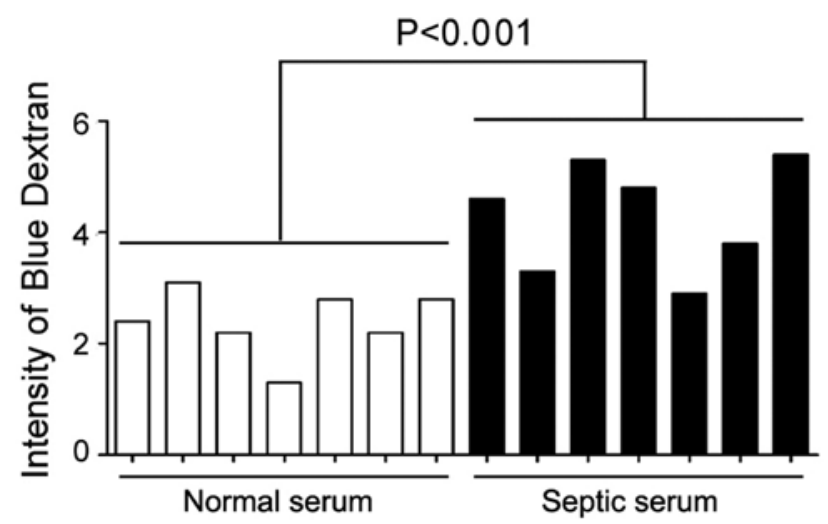

Figure 1. HUVEC monolayer was treated with septic serum or control serum for $12 \mathrm{~h}$. The integrity of the HUVEC monolayer was assayed by measuring blue dextran leakage. All data are representative of three independent experiments. ${ }^{* * *} \mathrm{P}<0.001$.

Since tight junction proteins, which seal the paracellular spaces between cells and contribute to endothelial barrier function, are frequently repressed during the EndMT process (10), we examined whether septic serum induced EndMT in endothelial cells. Markedly reduced expression of endothelial cell marker, CD31, and increased expression of mesenchymal cell markers, vimentin and $\alpha$-SMA, were detected by immunoblot analysis in the septic serum-treated HUVECs compared to the control cells (Fig. 2A). These observations were further supported by both loss of CD31 on the cell membrane and an increase in vimentin in the cytoplasm (Fig. 2B). Additionally, both migratory and invasive capability of HUVECs were elevated after treatment with septic serum, as shown by the 
A

B

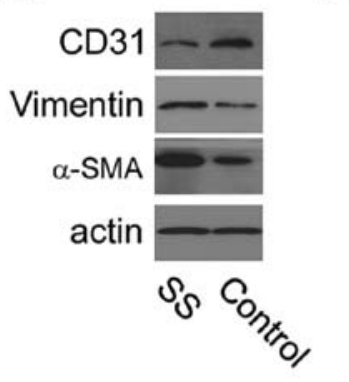

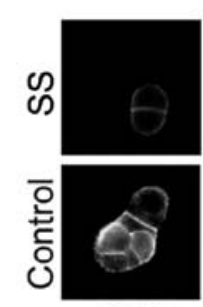

CD31

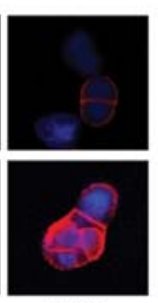

CD31 Hoechst

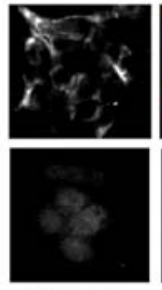

Vimentin

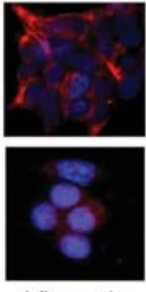

Vimentin

Hoechst

C
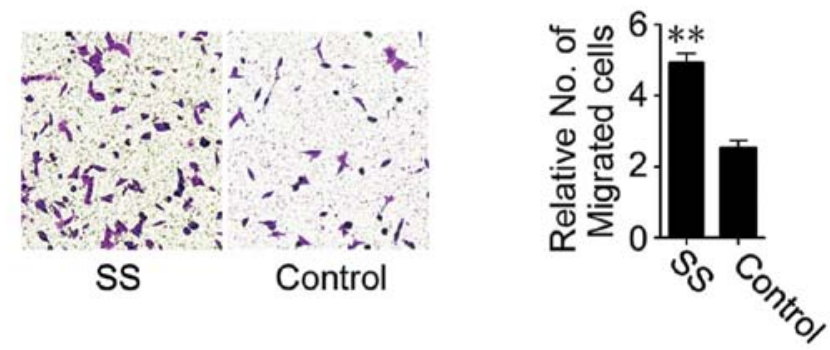

D
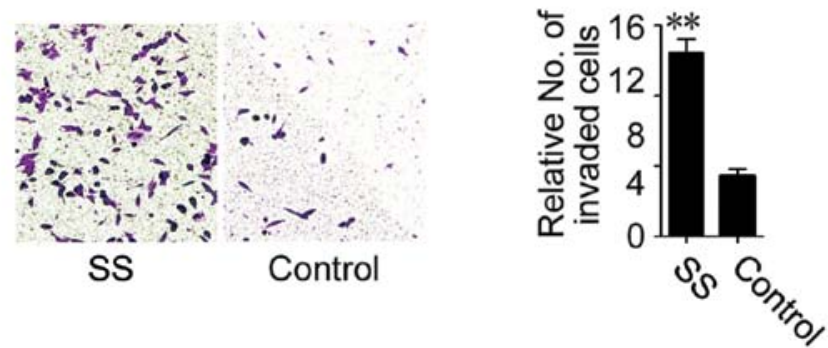

Figure 2. (A) HUVECs were treated with septic serum for $12 \mathrm{~h}$, and expression of vimentin, CD31 and $\alpha$-SMA was examined by immunoblot analysis. (B) Subcellular location of CD31 and vimentin was examined by immunocytofluorescence. The capability for cell migration and invasion was measured by (C) Transwell chamber migration and (D) Matrigel invasion assays. All data are representative of three independent experiments. ${ }^{* * *} \mathrm{P}<0.01$ compared to control. SS, septic serum.

Transwell chamber migration (Fig. 2C) and Matrigel invasion assays, respectively (Fig. 2D). These observations suggest that septic serum induces EndMT in endothelial cells.

Proteomic profiling of endothelial cells under septic stimuli. To further explore the mechanism of septic serum-induced EndMT in endothelial cells, 2-DE-based proteomic analysis was employed to profile the differentially expressed membrane proteins between septic serum-treated and untreated HUVECs. Representative 2-DE maps from seven parallel experiments are shown in Fig. 3A. As a result, a total of 29 protein spots were identified with an altered expression level. Among them, 18 spots were downregulated in response to septic serum, while 11 spots were upregulated. Fifteen representative altered spots were boxed and enlarged with the surrounding area (Fig. 3B).

The selected protein spots were then subjected to and identified by MS/MS analysis. As a result, all of the 29 spots were positively identified. The altered proteins were involved in diverse biological processes, including signaling transduction, cytoskeleton assembly, metabolism and adhesion, and the majority of these proteins were reported as membrane proteins according to ExPASy protein database. The detailed information of eight proteins with the most significant alterations are listed in Table II.
Validation of the altered proteins. To confirm the proteins exhibiting altered levels as revealed by proteomic analysis, the expression levels of four proteins with the most significant alterations, including caveolin-1, S100A4, $\alpha$-enolase and galectin-1, were examined by immunoblot analysis. S100A4 was found to be upregulated, while the level of caveolin-1, $\alpha$-enolase and galectin-1 were downregulated after septic serum treatment, which was in accordance with our proteomic analysis (Fig. 4).

Caveolin-1 is a negative regulator of EndMT during sepsis. Caveolin-1 was previously found to be involved in EMT in either normal epithelial cells or cancer cell lines (16-18). Therefore, we aimed to ascertain whether caveolin-1 has a role in septic serum-induced EndMT in HUVECs. To this goal, HUVECs were transfected with a caveolin-1-expressing vector prior to exposure to septic serum. As shown, septic serum-induced overexpression of vimentin and $\alpha$-SMA, and reduction of CD31 was substantially abolished upon increased caveolin-1 expression compared to mock control cells (Fig. 5A). These observations were further accompanied by markedly attenuated migratory and invasive capability of the HUVECs, as demonstrated by Transwell chamber migration (Fig. 5B) and Matrigel invasion assays (Fig. 5C), respectively. 


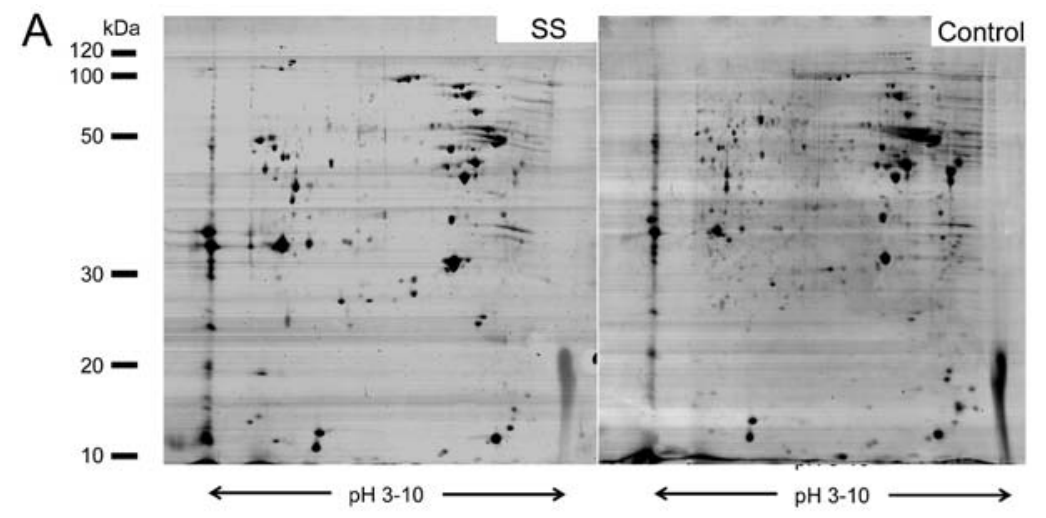

B
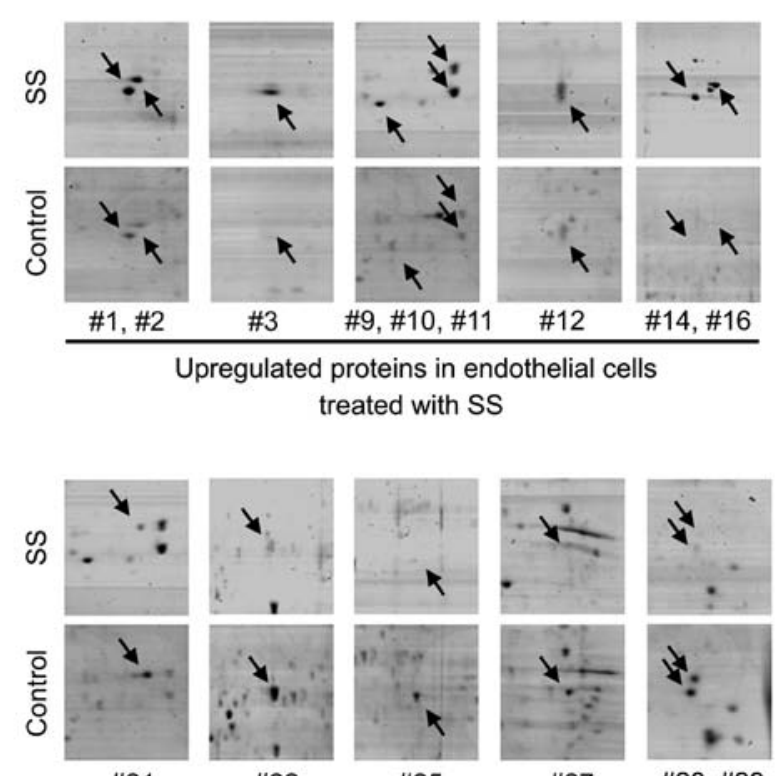

Downregulated proteins in endothelia cells treated with SS

Figure 3. HUVECs were treated with septic serum for $12 \mathrm{~h}$. Total protein was extracted, and then separated on pH 3.0-10.0 nonlinear IPG strips in the first dimension followed by $12 \%$ SDS-PAGE in the second dimension and visualized by CBB staining. (A) Approximately 1,300-1,400 protein spots were detected in a single 2-DE gel. (B) Fifteen representative protein spots exhibiting the most significant alteration are shown, and their corresponding spots are boxed and enlarged with the surrounding area. SS, septic serum.

Table II. Proteins demonstrating the most significant alteration in endothelial cells treated or untreated with septic serum.

\begin{tabular}{|c|c|c|c|c|c|c|}
\hline $\begin{array}{l}\text { Spot } \\
\text { no. }\end{array}$ & $\begin{array}{c}\text { Protein } \\
\text { description }\end{array}$ & $\begin{array}{l}\text { Gene } \\
\text { name }\end{array}$ & $\begin{array}{c}\text { Accession } \\
\text { no. }\end{array}$ & Function & Location & $\begin{array}{c}\text { Theoretical } \\
\text { Mr/pI }\end{array}$ \\
\hline 1 & $\begin{array}{l}\text { Chloride intracellular } \\
\text { channel protein } 1\end{array}$ & CLIC1 & O00299 & $\begin{array}{l}\text { Chloride ion } \\
\text { channel }\end{array}$ & Cell membrane & $26,791.54 / 5.09$ \\
\hline 2 & $\begin{array}{l}\text { Membrane-associated } \\
\text { progesterone receptor } \\
\text { component } 2\end{array}$ & PGRMC2 & O15173 & Signal transduction & Cell membrane & $23,818.45 / 4.76$ \\
\hline 3 & S100A4 & S100A4 & P26447 & Calcium ion binding & Cell membrane & $11,597.32 / 5.88$ \\
\hline 10 & Annexin A4 & ANXA4 & P09525 & $\begin{array}{l}\text { Membrane fusion } \\
\text { and exocytosis }\end{array}$ & Cell membrane & $36,088.00 / 5.84$ \\
\hline 16 & Hephaestin & HEPH & Q9BQS7 & Iron ion transport & Cell membrane & $127,792.48 / 5.64$ \\
\hline 21 & Caveolin-1 & CAV1 & Q2TNI1 & Lipid raft regulation & Lipid raft & $20,471.62 / 5.64$ \\
\hline 22 & $\alpha$-enolase & ENO1 & P06733 & Glycolysis & $\begin{array}{l}\text { Cytoplasm, } \\
\text { cell membrane }\end{array}$ & $47,037.77 / 6.99$ \\
\hline 28 & Galectin-1 & LGALS1 & P09382 & ECM remodeling & Extracellular space & $14,584.51 / 5.30$ \\
\hline
\end{tabular}


A

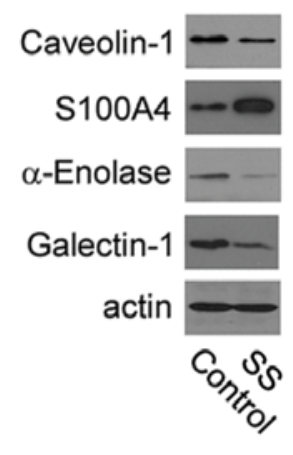

B

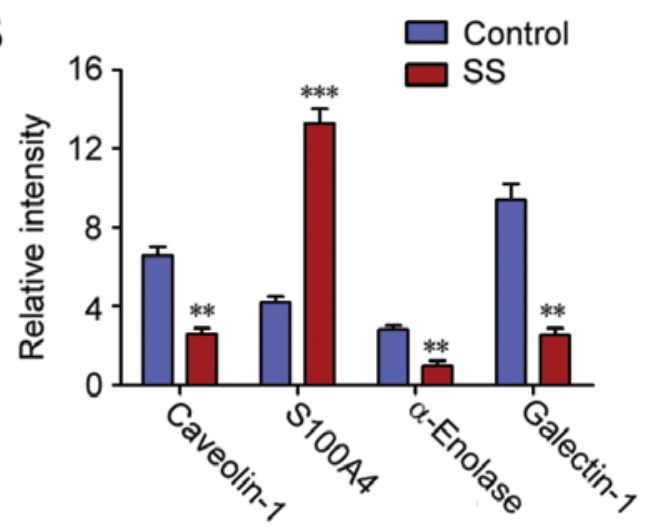

Figure 4. (A) Expression pattern of caveolin-1, S100A4, $\alpha$-enolase and galectin-1 were validated by western blotting. (B) Each blotting strip was determined by Quality-One software, and each value represents the average intensity of three parallel experimental runs. Pairs of total protein were normalized by $\beta$-actin. All data are representative of three independent experiments. ${ }^{* * *} \mathrm{P}<0.001 ;{ }^{* * *} \mathrm{P}<0.01$ compared with the control. SS, septic serum.

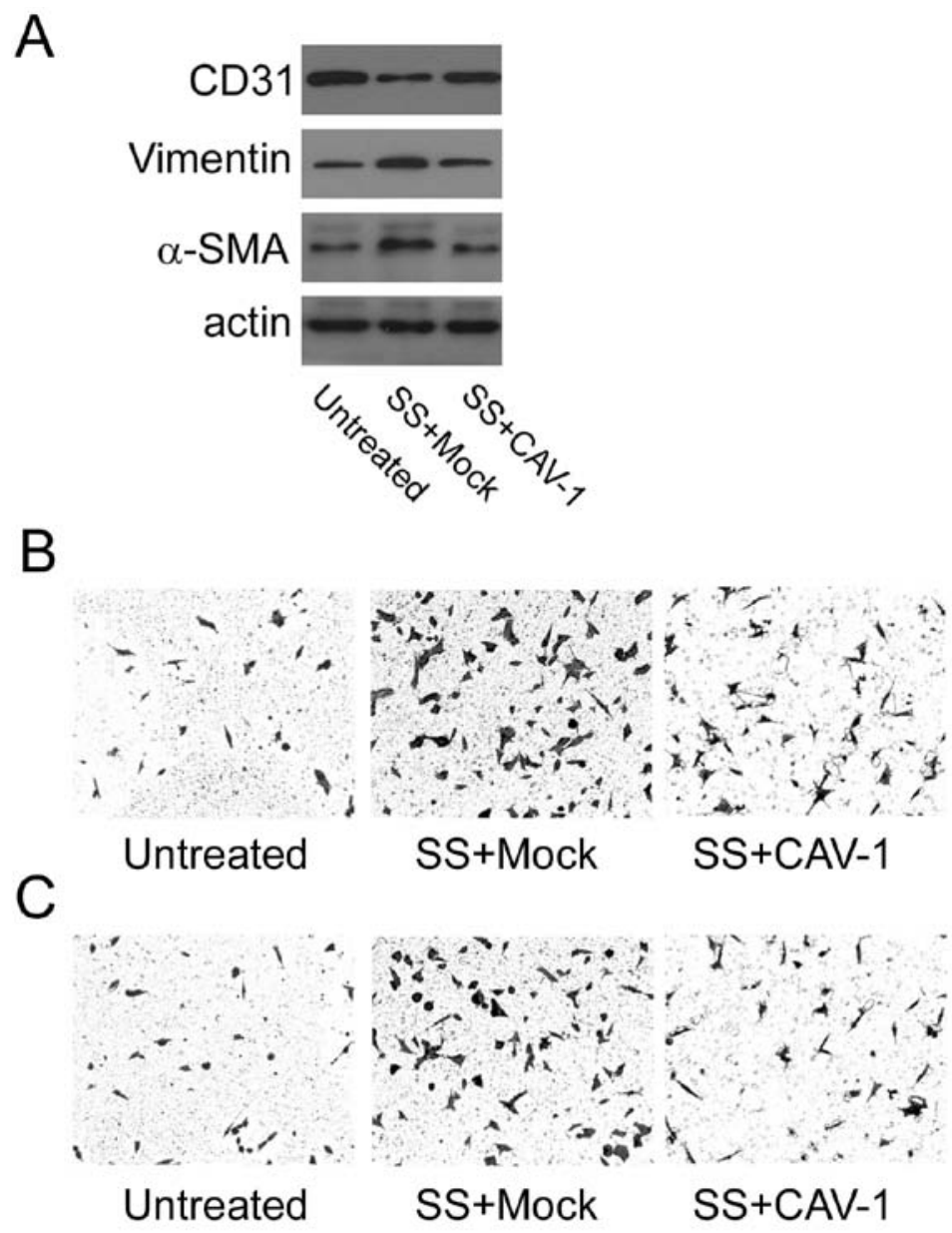

Figure 5. HUVECs were transfected with a caveolin-1-expressing vector or mock vector for $48 \mathrm{~h}$, and then treated with septic serum for $12 \mathrm{~h}$. (A) Expression of vimentin, CD31 and $\alpha$-SMA was examined by immunoblot analysis. Capability of cell migration and invasion was measured by (B) Transwell chamber migration and (C) Matrigel invasion assays. All data are representative of three independent experiments. SS, septic serum.

\section{Discussion}

Endothelial cells are involved in various aspects of vascular biology, such as maintenance of endothelial barrier integrity (4). Endothelial dysfunction which results from direct exposure to various types of stress, such as inflammatory mediators and microbial antigens, is universal in sepsis (19). In the present study, we evaluated endothelial dysfunction as a result of septic serum stimuli. We demonstrated that treatment with the serum derived from patients with sepsis induced a rapid decreased in the barrier integrity of a HUVEC monolayer. Notably, endothelial barrier dysfunction mediated by a 12-h exposure to septic serum was not due to increased cell death, since a detectable increase in HUVEC apoptosis was not 
observed until $36 \mathrm{~h}$ of incubation. We further demonstrated that septic serum triggered EndMT in HUVECs, as revealed by repression of CD31 and overexpression of $\alpha$-SMA and vimentin, which was accompanied by enhanced cell motility and invasiveness. This is the first study to report that endothelial cells undergo EndMT during sepsis. Furthermore, our data indicate that EndMT may contribute to septic stimuli-induced endothelial barrier dysfunction.

A small but increasing number of studies have shed light on the regulatory signaling network of EndMT. It is reported that Snail is required for TGF- $\beta$-induced EndMT of embryonic stem cell-derived endothelial cells (MESECs) and that transient expression of Snail induced the differentiation of MESECs into mural cells, whereas knockdown of Snail expression abrogated TGF- $\beta$-induced mural differentiation of MESECs (20). Inhibition of Smad3, a crucial node protein in TGF- $\beta$ signaling cascade, by a chemical antagonist, blocked EndMT in pancreatic microvascular endothelial cells, leading to a significant delay in the early development of streptozotocininduced diabetic nephropathy (21). Moreover, endothelin-1 has also been suggested as an EndMT inducer in cardiac fibrosis in diabetic hearts (22). In the present study, 2-DE-based proteomic approaches were employed to analyze the potential mediators of EndMT under sepsis, and 29 proteins with significant alterations were identified. Four proteins, including caveolon-1, S100A4, galectin-1 and $\alpha$-enolase, were selected and validated at the protein level.

Caveolin-1 is a 21- to 24-kDa cholesterol-binding membrane protein and is the major scaffolding component of caveolae (23). Abnormal expression of caveolin-1 has long been implicated during EMT, which is a process similar to EndMT. It is reported that downregulation of caveolin-1 by chronic exposure to EGF leads to the loss of E-cadherin, and enhanced epidermoid carcinoma cell invasion (16). Consistently, it has been shown that caveolin-1 promotes pancreatic cancer cell differentiation by suppression of EMT via upregulation of E-cadherin expression (17). Nevertheless, an enhanced level of caveolin-1 was observed in both human embryonic carcinoma cell line NT2/D1 and normal mouse mammary epithelial cell line NMuMG following the induction of EMT. Upregulation of caveolin-1 in these two cell models was probably mediated by activation of focal adhesion kinase (FAK) and Src, two known tyrosine kinases involved in EMT (18). Although both EMT and EndMT give rise to cells that have a mesenchymal phenotype (24), the role of caveolin-1 in EndMT is still uncharacterized. Based on our data, exogenous expression of caveolin-1 in HUVECs markedly blocked septic serum-induced EndMT, by retrieving expression of CD31 and repressing expression of both $\alpha$-SMA and vimentin. In correlation, increased migratory and invasive capability of HUVECs was markedly attenuated upon caveolin-1 expression. Therefore, it is reasonable to infer that caveolin-1 functions as a negative regulator of EndMT in response to septic stimuli.

In summary, we reported that exposure to septic serum induced EndMT and conferred a migratory and invasive phenotype on endothelial cells. Furthermore, we profiled the proteomic alterations in endothelial cells undergoing septic stimuli, and 29 proteins with differentially expressed levels were identified. Finally, we demonstrated that exogenous expression of caveolin-1 attenuated septic serum-induced EndMT as well as migratory and invasive capability in HUVECs. The present study may lead to a better understanding of the biological role of endothelial cells in the pathophysiology of sepsis.

\section{Acknowledgements}

This study was supported by the Department of Public Health of Sichuan Province (grants 080326).

\section{References}

1. Cornell TT, Rodenhouse P, Cai Q, Sun L and Shanley TP: Mitogen-activated protein kinase phosphatase 2 regulates the inflammatory response in sepsis. Infect Immun 78: 2868-2876, 2010.

2. Akrout N, Sharshar T and Annane D: Mechanisms of brain signaling during sepsis. Curr Neuropharmacol 7: 296-301, 2009.

3. Liu SF and Malik AB: NF-kappa B activation as a pathological mechanism of septic shock and inflammation. Am J Physiol Lung Cell Mol Physiol 290: L622-L645, 2006.

4. Shapiro NI, Schuetz P, Yano K, et al: The association of endothelial cell signaling, severity of illness, and organ dysfunction in sepsis. Crit Care 14: R182, 2010.

5. Aird WC: The role of the endothelium in severe sepsis and multiple organ dysfunction syndrome. Blood 101: 3765-3777, 2003.

6. Lee WL and Slutsky AS: Sepsis and endothelial permeability. N Engl J Med 363: 689-691, 2010.

7. Garcia J, Sandi MJ, Cordelier P, et al: Tie1 deficiency induces endothelial-mesenchymal transition. EMBO Rep 13: 431-439, 2012.

8. Yoshimatsu Y and Watabe T: Roles of TGF- $\beta$ signals in endothelial-mesenchymal transition during cardiac fibrosis. Int J Inflam 2011: 724080, 2011.

9. van Meeteren LA and ten Dijke P: Regulation of endothelial cell plasticity by TGF- $\beta$. Cell Tissue Res 347: 177-186, 2012.

10. Nagasawa K, Chiba H, Fujita H, et al: Possible involvement of gap junctions in the barrier function of tight junctions of brain and lung endothelial cells. J Cell Physiol 208: 123-132, 2006.

11. Zeisberg EM, Potenta SE, Sugimoto H, Zeisberg $M$ and Kalluri R: Fibroblasts in kidney fibrosis emerge via endothelialto-mesenchymal transition. J Am Soc Nephrol 19: 2282-2287, 2008.

12. Tanjore $\mathrm{H}, \mathrm{Xu} \mathrm{XC}$, Polosukhin VV, et al: Contribution of epithelial-derived fibroblasts to bleomycin-induced lung fibrosis. Am J Respir Crit Care Med 180: 657-665, 2009.

13. Wu CC and Yates JR III: The application of mass spectrometry to membrane proteomics. Nat Biotechnol 21: 262-267, 2003.

14. Xiong P, Li Y, Tang Y and Chen H: Proteomic analyses of Sirt1mediated cisplatin resistance in OSCC cell line. Protein $\mathrm{J} 30$ : 499-508, 2011.

15. Tsao N, Hsu HP, Wu CM, Liu CC and Lei HY: Tumour necrosis factor-alpha causes an increase in blood-brain barrier permeability during sepsis. J Med Microbiol 50: 812-821, 2001.

16. Lu Z, Ghosh S, Wang Z and Hunter T: Downregulation of caveolin-1 function by EGF leads to the loss of E-cadherin, increased transcriptional activity of $\beta$-catenin, and enhanced tumor cell invasion. Cancer Cell 4: 499-515, 2003.

17. Salem AF, Bonuccelli G, Bevilacqua $G$, et al: Caveolin-1 promotes pancreatic cancer cell differentiation and restores membranous E-cadherin via suppression of the epithelial-mesenchymal transition. Cell Cycle 10: 3692-3700, 2011.

18. Bailey KM and Liu J: Caveolin-1 up-regulation during epithelial to mesenchymal transition is mediated by focal adhesion kinase. J Biol Chem 283: 13714-13724, 2008.

19. Schuetz P, Jones AE, Aird WC and Shapiro NI: Endothelial cell activation in emergency department patients with sepsis-related and non-sepsis-related hypotension. Shock 36: 104-108, 2011.

20. Kokudo T, Suzuki Y, Yoshimatsu Y, Yamazaki T, Watabe T and Miyazono K: Snail is required for TGFbeta-induced endothelial-mesenchymal transition of embryonic stem cell-derived endothelial cells. J Cell Sci 121: 3317-3324, 2008.

21. Li J, Qu X, Yao J, et al: Blockade of endothelial-mesenchymal transition by a Smad3 inhibitor delays the early development of streptozotocin-induced diabetic nephropathy. Diabetes 59: 2612-2624, 2010 
22. Widyantoro B, Emoto N, Nakayama K, et al: Endothelial cellderived endothelin-1 promotes cardiac fibrosis in diabetic hearts through stimulation of endothelial-to-mesenchymal transition. Circulation 121: 2407-2418, 2010.

23. Zhu Y, Liao HL, Wang N, et al: Lipoprotein promotes caveolin-1 and ras translocation to caveolae: role of cholesterol in endothelial signaling. Arterioscler Thromb Vasc Biol 20: 2465-2470, 2000 .
24. Potenta S, Zeisberg E and Kalluri R: The role of endothelial-tomesenchymal transition in cancer progression. Br J Cancer 99: 1375-1379, 2008

25. Bone RC, Balk RA, Cerra FB, et al: Definitions for sepsis and organ failure and guidelines for the use of innovative therapies in sepsis. The ACCP/SCCM Consensus Conference Committee. American College of Chest Physicians/Society of Critical Care Medicine. Chest 101: 1644-1655, 1992. 